\title{
Cardiac Thrombus Does Not Increase Post-Alteplase Microbembolic Events: Machine Learning and Propensity Score Augmented Cohort Analysis of 434 Ischemic Stroke Patients with Bayesian Simulated Analysis of 100,000 Patients
}

\author{
Danielle Stone $^{1 *}$, Dominique Monlezun ${ }^{2 *}$, James Stone ${ }^{1 *}$, Humayun Naqvi ${ }^{1}$, Amanda Jagolino- \\ Cole $^{3}$ and Francisco Fuentes ${ }^{1}$
}

${ }^{1}$ Division of Cardiovascular Medicine, University of Texas Health Sciences Center at Houston, Houston, TX, USA

${ }^{2}$ Department of Cardiology, University of Texas M.D. Anderson Cancer Center, Houston, TX, USA

${ }^{3}$ Department of Neurology, University of Texas Health Sciences Center at Houston, Houston, TX, USA

*Corresponding author: Danielle Stone, MD, Division of Cardiovascular Medicine, Department of Internal Medicine, McGovern Medical School, 6431 Fannin Street, Houston, Texas 77030, USA

Contributions: *Co-first authors contributed equally to this manuscript

To Cite This Article: Danielle S, Dominique M, James S, Humayun N, A Jagolino-Cole, et al., Cardiac Thrombus Does Not Increase PostAlteplase Microbembolic Events: Machine Learning and Propensity Score Augmented Cohort Analysis of 434 Ischemic Stroke Patients with Bayesian Simulated Analysis of 100,000 Patients. 2020 - 8(3). AJBSR.MS.ID.001266. DOI: 10.34297/AJBSR.2020.08.001266

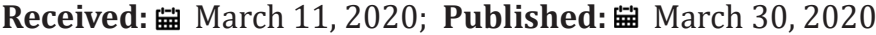

\section{Background}

Stroke kills more patients globally than any other disease outside of ischemic heart disease, with prompt systemic thrombolysis being the standard of care for the majority of strokes (acute ischemic stroke [AIS]) [1]. Its benefit over risk ratio is so notable that recent randomized trials were conducted to determine if the treatment window could be extended. Yet, there is a concern for embolic complications for patients who receive tissue plasminogen activator (tPA) in the setting of cardiac thrombi [2], with an estimated nearly 500,000 stroke patients annually with cardiac thrombus who may be at risk for such post-tPA complications $[3,4]$. But there are no adequately powered (let alone randomized) studies assessing post-thrombolysis embolization in patients with cardiac thrombus and AIS [5]. Our study is the first known to apply state-of-the-art machine learning augmentation of causal inference propensity score analysis (backed by Bayesian statistics to simulate a trial of 100,000 subjects) to evaluate if thrombolysis increases microembolic events (MEs) among cardiac thrombus patients with AIS.

\section{Methods}

This retrospective cohort study assessed post-tPA ME as the primary outcome and length of stay (LOS) in days and poor functional outcome at discharge ( $>2$ modified Rankin score) as secondary outcomes. Inclusion criteria were cardioembolic AIS patients presenting sequentially at a single quaternary academic medical center in Houston, Texas, USA, from 01/01/2012$02 / 31 / 2017$, with exclusion criteria being no recorded presence or absence of ME and cardiac thrombus.

A. Propensity score adjusted penalized multivariable logistic regression was performed (adjusting for age, admission NIHSS, and the likelihood of receiving tPA [additional variables were excluded based upon the below regression diagnostics]) including the interaction term of tPA and cardiac thrombus as a model variable. Stratification by tPA was conducted with margins probabilities to calculate average adjusted predictions. The final models were optimized for performance based on standard regression diagnostic tests in addition to comparison to a machine learning-based backward propagation neural network.

B. Augmented inverse propensity weighted (AIPW) analysis was then conducted with the same model parameters as [1] to compare the results to [1]. 
C. Finally, Bayesian regression was performed among tPA patients using Metropolis-Hastings algorithm to simulate a sample size of 100,000 patients (since power analysis indicated that 8,351 subjects would be required to detect a $50 \%$ difference in ME by cardiac thrombus). Statistical significance was set at two-tailed p-value $<0.05$. All statistical analyses were performed using STATA 14.2 (STATACorp, College Station, Texas, USA), and machine learning analysis was conducted using Java 9 (Oracle, Redwood Chores, California, USA).

\section{Results}

Among the 434 patients meeting criteria, the mean age was 65 (11 SD), the mean admission NIHSS was 12 (SD 9), 1.84\% had MEs among whom 62.50\% received tPA, and 19 (4.38\%) had cardiac thrombus on TEE among whom $4.68 \%$ received tPA and none regardless of receiving tPA had MEs (Table 1). In propensity score adjusted multivariable regression, tPA for cardiac thrombus patients did not significantly increase ME (OR 7.31, 95\%CI 0.32169.29; $\mathrm{p}=0.215$ ), LOS (beta $-0.99,95 \% \mathrm{CI}-7.50-5.52 ; \mathrm{p}=0.766$ ), nor poor functional outcome (OR 0.53, 95\% CI 0.11-2.54; $\mathrm{p}=0.424$ ). Stratified regression among tPA subjects produced similarly nonsignificant results for cardiac thrombus and ME (reducing its risk by $52.27 \%$ ), LOS, and poor functional outcome. AIPW analysis demonstrated no significant association between tPA and ME. Bayesian regression similarly demonstrated that cardiac thrombus did not increase ME (reducing its probability by nearly $24.48 \%$ ).

\begin{tabular}{|c|c|c|c|c|}
\hline \multirow[t]{2}{*}{ Variable } & \multirow{2}{*}{$\begin{array}{c}\text { Sample } \\
N=434\end{array}$} & \multicolumn{2}{|c|}{ Microembolic Events } & \multirow[t]{2}{*}{ P-value } \\
\hline & & No $(n=426,98.16 \%)$ & Yes $(\mathrm{n}=8,1.84 \%)$ & \\
\hline \multicolumn{5}{|l|}{ Demographic } \\
\hline Age, mean (SD) & 64.89 (11.19) & $64.94(11.23)$ & $62.13(9.17)$ & 0.481 \\
\hline \multicolumn{5}{|l|}{ Comorbidities, no. (\%) } \\
\hline Diabetes & $114(26.27)$ & $111(26.06)$ & $3(37.50)$ & 0.438 \\
\hline Hypertension & $230(53.00)$ & 227 (53.29) & $3(37.50)$ & 0.483 \\
\hline CAD & $44(10.16)$ & $44(10.35)$ & $0(0.00)$ & 1.000 \\
\hline PVD & $3(0.69)$ & $3(0.70)$ & $0(0.00)$ & 1.000 \\
\hline CABG/Stent & $15(3.46)$ & $15(3.53)$ & $0(0.00)$ & 1.000 \\
\hline Atrial fibrillation & $135(31.11)$ & $132(30.99)$ & $3(37.50)$ & 0.708 \\
\hline Carotid stenosis & $2(0.46)$ & $2(0.47)$ & $0(0.00)$ & 1.000 \\
\hline CEA/Stent & $2(0.46)$ & $2(0.47)$ & $0(0.00)$ & 1.000 \\
\hline CHF & 50 (11.52) & $50(11.74)$ & $0(0.00)$ & 0.605 \\
\hline Cancer & $29(6.68)$ & $29(6.81)$ & $0(0.00)$ & 1.000 \\
\hline Coagulation disorder & $6(1.38)$ & $6(1.41)$ & $0(0.00)$ & 1.000 \\
\hline Prior DVT/PE & $0(0.00)$ & $0(0.00)$ & $0(0.00)$ & 1.000 \\
\hline Prior CVA & $81(18.66)$ & 69 (18.54) & $2(25.00)$ & 0.647 \\
\hline \multicolumn{5}{|l|}{ Prior MI } \\
\hline \multicolumn{5}{|l|}{ Cerebrovascular event, no. (\%) } \\
\hline tPA given & $171(39.40)$ & $166(38.97)$ & $5(62.50)$ & 0.273 \\
\hline \multicolumn{5}{|l|}{ NIHSS, mean (SD) } \\
\hline Admission & $11.70(8.81)$ & $11.58(8.82)$ & $17.88(5.96)$ & 0.045 \\
\hline Post-tPA & $9.52(8.97)$ & $9.59(9.04)$ & $6.33(3.51)$ & 0.536 \\
\hline Admit to tPA, min median (range) & $39(23-57)$ & $37(23-57)$ & $41(39-78)$ & 0.280 \\
\hline Cardiac thrombus & $19(4.38)$ & $19(4.46)$ & $0(0.00)$ & 1.000 \\
\hline \multicolumn{5}{|l|}{ Outcomes, median (range) } \\
\hline LOS, days & $5(3-9)$ & $5(3-9)$ & $9(5-12)$ & 0.202 \\
\hline \multicolumn{5}{|l|}{ Modified Rankin score } \\
\hline Discharge & $4(2-5)$ & $4(2-5)$ & $4(3-6)$ & 0.147 \\
\hline Poor $(>2)$ & $238(72.78)$ & $230(72.10)$ & $8(100.00)$ & 0.113 \\
\hline 90 days & $4(2-6)$ & $4(2-6)$ & $5(3-6)$ & 0.514 \\
\hline Poor $(>2)$ & $123(69.10)$ & $120(68.97)$ & $3(75.00)$ & 1.000 \\
\hline Complications & 114 (26.27) & $111(26.06)$ & $3(37.50)$ & 0.438 \\
\hline
\end{tabular}

*SD, standard deviation; CAD, coronary artery disease; MI, myocardial infarction; PVD, peripheral vascular disease; CABG, coronary artery bypass grafting; CEA, carotid endarterectomy; CHF, congestive heart failure; DVT, deep vein thrombosis; PE, pulmonary embolus; CVA, cerebrovascular accident; tPA, tissue plasminogen activator; min, minutes; LOS, length of stay. 


\section{Discussion}

This is the largest known study of post-thrombolysis ME and cardiac thrombus among AIS subjects, and the first to use robust causal inference statistics supported by machine learning and Bayesian statistics. Our analysis suggests that cardiac thrombus does not increase post-thrombolysis ME in AIS, a finding supported by a Bayesian analysis modelling 100,000 patients. Our study does have limitations including a non-randomized design using a single center dataset with a rare primary outcome. Although large randomized controlled trials are needed to confirm these findings, it is unlikely that such trials will occur (given the expected enrollment challenges), leaving this study as a potentially helpful addition to the existing literature to guide clinicians in such a challenging scenario.

\section{References}

1. Emberson J, Lees KR, Lyden P, Blackwell L, Albers G, et al. (2014) Effect of treatment delay, age, and stroke severity on the effects of intravenous thrombolysis with alteplase for acute ischaemic stroke: a meta-analysis of individual patient data from randomised trials. Lancet 384(9958): 1929-1935.

2. Bautista RE (1995) Embolic stroke following thrombolytic therapy for myocardial infarction in a patient with preexisting ventricular thrombi. Stroke 26(2): 324-325.

3. Bogousslavsky J, Cachin C, Regli F, Despland PA, Van Melle G, et al. (1991) Lausanne Stroke Registry Group. Cardiac sources of embolism and cerebral infarction. Clinical consequences and vascular concomitants: The Lausanne Stroke Registry. Neurology 41(6): 855-859.

4. Katan M, Luft A (2018) Global burden of stroke. Semin Neurol 38(2): 208211.

5. Derex L, Nighoghossian N, Perinetti M, Honnorat J, Trouillas P (2001) Thrombolytic therapy in acute ischemic stroke patients with cardiac thrombus. Neurology 57(11): 2122-2125. 